\title{
Técnicas de Geração de Superfícies Topográficas Suaves Através de Malhas Triangulares
}

F. S. de SOUSA ${ }^{1}$, M. MENEGUETTE Jr², Departamento de Matemática, Faculdade de Ciências e Tecnologia, UNESP, Cx.P. 957, 19060-900 Presidente Prudente, SP, Brasil.

Resumo. O objetivo deste trabalho é encontrar métodos de suavizar uma malha triangular de pontos do espaço euclidiano, a fim de visualizar a superfície que melhor se ajusta a esta malha. Para isso, foi necessário um estudo sobre algumas parametrizações utilizadas nas reconstruções de superfícies, tais como as de Coons, Bézier e B-Spline, resultando em eficientes algoritmos para a determinação de tais superfícies.

\section{Introdução}

A representação de superfícies topográficas no computador pode ser feita através de malhas poligonais, de modo que as faces desta malha sejam formadas por triângulos. Estas superfícies são chamadas de malhas triangulares. Este tipo de representação é extremamente fácil e evita, por exemplo, uma triangulação de Delaunay, reduzindo consideravelmente os custos computacionais de visualização.

Neste sentido, buscamos, neste trabalho, algumas técnicas de reconstrução de superfícies suaves a partir de uma malha triangular, que nada mais são do que métodos de ajustes de superfícies, nos quais destacamos superfícies de Bézier e B-Spline.

Há ainda uma outra forma de parametrização destas superfícies, chamadas superfícies de Coons, que utilizam dados não intuitivos, como curvas de contorno e vetores tangente, dificultando sua utilização.

\section{Superfícies de Coons}

Superfícies de Coons nescessitam da descrição das curvas de contorno e/ou vetores tangentes e mistos das mesmas, dependendo do grau de precisão requerido na reconstrução da superfície. Esta será obtida através de funções de combinação, que irão ditar o formato da superfície.

Serão descritos, a seguir, dois tipos de superfícies de Coons, que são classificados a partir de suas funções de combinação: a linear e a bicúbica.

\footnotetext{
${ }^{1}$ fsimeoni@icmc.sc.usp.br

2 messias@prudente.unesp.br
} 


\subsection{Superfície de Coons linear}

Uma superfície de Coons linear é obtida através das quatro curvas de controle $P(u, 0), P(u, 1), P(0, w)$ e $P(1, w)$, supostas conhecidas, e de uma função de combinação bilinear usada para determinar os pontos sobre a superfície.

Uma primeira tentativa de representar parametricamente tal superfície é tomar a combinação bilinear das curvas de contorno nas direções de $u$ e $w$, ou seja,

$$
Q(u, w)=P(u, 0)(1-w)+P(u, 1) w+P(0, w)(1-u)+P(1, w) u .
$$

No entanto, examinando o resultado nos cantos da superfície, por exemplo em $Q(0,0)$, tem-se

$$
Q(0,0)=P(0,0)+P(0,0)=2 P(0,0) .
$$

E sobre os caminhos, por exemplo em $Q(0, w)$, tem-se

$$
Q(0, w)=P(0,0)(1-w)+P(0,1) w+P(0, w) .
$$

Nenhum destes resultados correspondem aos dados originais da superfície como visto. Este problema ocorre porque os pontos extremos da superfície (cantos) são contados duas vezes, por exemplo, o ponto $P(0,0)$ está contido nas curvas de contorno $P(u, 0)$ e $P(0, w)$.

O resultado correto é obtido subtraindo-se os excessos dados pelos pontos extremos. Isto resulta em

$$
\begin{aligned}
Q(u, w)= & P(u, 0)(1-w)+P(u, 1) w+P(0, w)(1-u) \\
& +P(1, w) u-P(0,0)(1-u)(1-w) \\
& -P(0,1)(1-u) w-P(1,0) u(1-w)-P(1,1) u w
\end{aligned}
$$

Assim, nos pontos extremos da superfície,

$$
\begin{aligned}
& Q(0,0)=P(0,0), \\
& Q(0,1)=P(0,1), \\
& Q(1,0)=P(1,0), \\
& Q(1,1)=P(1,1) .
\end{aligned}
$$

E, sobre as curvas de contorno,

$$
\begin{aligned}
Q(u, 0) & =P(u, 0), \\
Q(u, 1) & =P(u, 1), \\
Q(0, w) & =P(0, w), \\
Q(1, w) & =P(1, w) .
\end{aligned}
$$

Colocando (2.1) na forma matricial, obtém-se

$$
\begin{aligned}
& Q(u, w)=\left[\begin{array}{ll}
1-u & u
\end{array}\right]\left[\begin{array}{l}
P(0, w) \\
P(1, w)
\end{array}\right]+\left[\begin{array}{ll}
P(u, 0) & P(u, 1)
\end{array}\right]\left[\begin{array}{c}
1-w \\
w
\end{array}\right] \\
& -\left[\begin{array}{ll}
1-u & u
\end{array}\right]\left[\begin{array}{ll}
P(0,0) & P(0,1) \\
P(1,0) & P(1,1)
\end{array}\right]\left[\begin{array}{c}
1-w \\
w
\end{array}\right] \text {, }
\end{aligned}
$$


ou ainda, mais resumidamente,

$$
Q(u, w)=\left[\begin{array}{lll}
1-u & u & 1
\end{array}\right]\left[\begin{array}{ccc}
-P(0,0) & -P(0,1) & P(0, w) \\
-P(1,0) & -P(1,1) & P(1, w) \\
P(u, 0) & P(u, 1) & 0
\end{array}\right]\left[\begin{array}{c}
1-w \\
w \\
1
\end{array}\right] .
$$

As funções $(1-u), u,(1-w)$ e $w$ são chamadas funções de combinação, pois elas combinam as curvas de contorno para produzir o formato interno da superfície.

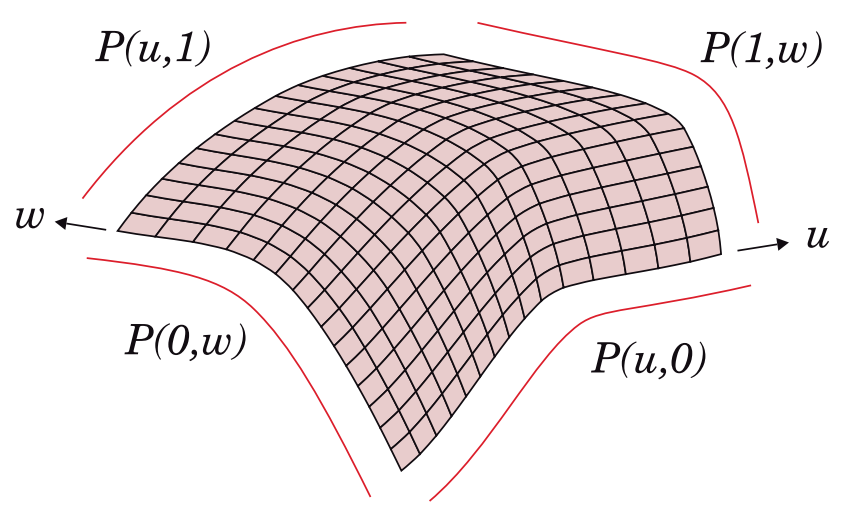

Figura 1: Superfície de Coons linear.

\subsection{Superfície de Coons bicúbica}

A superfície de Coons linear, apesar de fácil implementação, não tem flexibilidade suficiente para muitas aplicações.

As descrições de superfícies discutidas nesta e nas seções seguintes, trazem esta requerida flexibilidade usando polinômios de alto grau, tanto para as curvas de contorno quanto para as funções de combinação que descrevem o formato da superfície.

Superfícies de Coons bicúbicas utilizam splines cúbicas (ver mais detalhes em [3] e [5]) em todas as quatro curvas de contorno e funções de combinação, também cúbicas, são usadas para definir o formato da superfície.

Assim, cada curva de contorno tem a seguinte forma geral:

$$
P(t)=B_{1}+B_{2} t+B_{3} t^{2}+B_{4} t^{3}, \quad 0 \leq t \leq 1 .
$$

Para uma spline cúbica simples, onde são conhecidos os vetores de posição e os vetores tangente, cada uma das quatro curvas de contorno $P(u, 0), P(u, 1), P(0, w)$ e $P(1, w)$ são dadas por:

$$
P(t)=[T][N][G]=\left[\begin{array}{llll}
t^{3} & t^{2} & t & 1
\end{array}\right]\left[\begin{array}{cccc}
2 & -2 & 1 & 1 \\
-3 & 3 & -2 & -1 \\
0 & 0 & 1 & 0 \\
1 & 0 & 0 & 0
\end{array}\right]\left[\begin{array}{c}
P_{1} \\
P_{2} \\
P_{1}^{\prime} \\
P_{2}^{\prime}
\end{array}\right]
$$


onde $0 \leq t \leq 1$ e assume os valores de $u$ ou $w$, conforme for apropriado. Aqui, $P_{1}$, $P_{2}, P_{1}^{\prime}$ e $P_{2}^{\prime}$ são os vetores de posição e os vetores tangente dos extremos de cada curva considerada.

As funções de combinação são idênticas para as direções $u$ e $w$, tendo sua forma geral dada por:

$$
[F]=\left[\begin{array}{llll}
F_{1}(t) & F_{2}(t) & F_{3}(t) & F_{4}(t)
\end{array}\right]=[T][N],
$$

ou seja,

$$
[F]=\left[\begin{array}{llll}
t^{3} & t^{2} & t & 1
\end{array}\right]\left[\begin{array}{cccc}
2 & -2 & 1 & 1 \\
-3 & 3 & -2 & -1 \\
0 & 0 & 1 & 0 \\
1 & 0 & 0 & 0
\end{array}\right]
$$

Desenvolvendo este produto, obtemos

$$
\begin{aligned}
& F_{1}(t)=2 t^{3}-3 t^{2}+1 \\
& F_{2}(t)=-2 t^{3}+3 t^{2} \\
& F_{3}(t)=t^{3}-2 t^{2}+t \\
& F_{4}(t)=t^{3}-t^{2}
\end{aligned}
$$

onde $t$ assume o valor de $u$ ou $w$ conforme for apropriado.

Com isso, uma superfícies de Coons bicúbica é dada por:

$$
\begin{aligned}
Q(u, w)= & {\left[\begin{array}{lllll}
F_{1}(u) & F_{2}(u) & F_{3}(u) & F_{4}(u)
\end{array}\right] } \\
& \times\left[\begin{array}{cccc}
P(0,0) & P(0,1) & P_{w}(0,0) & P_{w}(0,1) \\
P(1,0) & P(1,1) & P_{w}(1,0) & P_{w}(1,1) \\
P_{u}(0,0) & P_{u}(0,1) & P_{u w}(0,0) & P_{u w}(0,1) \\
P_{u}(1,0) & P_{u}(1,1) & P_{u w}(1,0) & P_{u w}(1,1)
\end{array}\right]\left[\begin{array}{l}
F_{1}(w) \\
F_{2}(w) \\
F_{3}(w) \\
F_{4}(w)
\end{array}\right],
\end{aligned}
$$

para $0 \leq u \leq 1$ e $0 \leq w \leq 1$. Usando a equação (2.5), pode-se escrever (2.7) de uma forma mais compacta:

$$
Q(u, w)=[U][N][P][N]^{T}[W],
$$

onde $[U]=\left[\begin{array}{llll}u^{3} & u^{2} & u & 1\end{array}\right]$ e $[W]=\left[\begin{array}{llll}w^{3} & w^{2} & w & 1\end{array}\right]^{T}$.

A matriz $[P]$, dada pela equação (2.7), contém todas as informações requeridas para gerar uma superfície de Coons bicúbica. As submatrizes $2 \times 2$ em cada canto de $[P]$ contém:

$$
[P]=\left[\begin{array}{c|c}
\text { vetores extremos } & \text { vetores tangente } \\
(\text { de canto }) & (\text { direção } w) \\
\hline \text { vetores tangente } & \text { vetores de derivadas } \\
(\text { direção } u) & \text { mistas }(u \text { e } w)
\end{array}\right]
$$

Assim, uma superfície bicúbica é definida pelos quatro vetores de posição (cantos), oito vetores tangente (dois em cada canto) quatro vetores de derivadas mistas, também nos cantos, e por quatro funções de combinação cúbicas $F_{1}, F_{2}, F_{3}$ e $F_{4}$. 


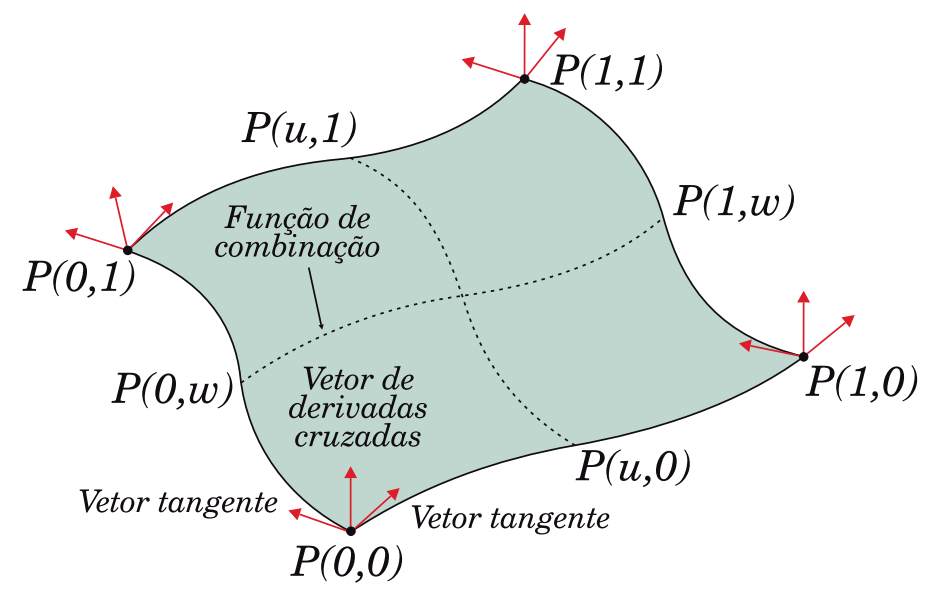

Figura 2: Geometria de uma superfície de Coons bicúbica.

As derivadas paramétricas em qualquer ponto da superfície são obtidas derivandose formalmente a equação (2.8), resultando:

$$
\begin{aligned}
Q_{u}(u, w) & =\frac{\partial Q}{\partial u}(u, w)=\left[U^{\prime}\right][N][P][N]^{T}[W], \\
Q_{w}(u, w) & =\frac{\partial Q}{\partial w}(u, w)=[U][N][P][N]^{T}\left[W^{\prime}\right], \\
Q_{u w}(u, w) & =\frac{\partial^{2} Q}{\partial u \partial w}(u, w)=\left[U^{\prime}\right][N][P][N]^{T}\left[W^{\prime}\right], \\
Q_{u u}(u, w) & =\frac{\partial^{2} Q}{\partial u^{2}}(u, w)=\left[U^{\prime \prime}\right][N][P][N]^{T}[W], \\
Q_{w w}(u, w) & =\frac{\partial^{2} Q}{\partial w^{2}}(u, w)=[U][N][P][N]^{T}\left[W^{\prime \prime}\right],
\end{aligned}
$$

onde

$$
\begin{aligned}
{\left[U^{\prime}\right] } & =\left[\begin{array}{llll}
3 u^{2} & 2 u & 1 & 0
\end{array}\right] \\
{\left[W^{\prime}\right] } & =\left[\begin{array}{llll}
3 w^{2} & 2 w & 1 & 0
\end{array}\right]^{T}, \\
{\left[U^{\prime \prime}\right] } & =\left[\begin{array}{llll}
6 u & 2 & 0 & 0
\end{array}\right] \\
{\left[W^{\prime \prime}\right] } & =\left[\begin{array}{llll}
6 w & 2 & 0 & 0
\end{array}\right]^{T} .
\end{aligned}
$$

O vetor normal à superfície, importante para determinação de superfícies ocultas e para aplicação em modelos de iluminação, é facilmente calculado pelo produto vetorial:

$$
\vec{n}=Q_{u} \times Q_{w} .
$$

Apesar de muito flexível e de uma descrição precisa, este tipo de superfície é pouco aplicável devido a grande quantidade de informações requeridas pelo usuário para gerar a superfície. No entanto, com esta descrição matemática pode-se obter um eficiente algoritmo. 


\section{Superfícies de Bézier}

Superfícies de Coons bicúbicas consistem em uma flexível e poderosa ferramenta no desenvolvimento de superfícies. No entanto, devido ao uso de splines cúbicas, sua utilização é prejudicada pela necessidade de especificar informações matemáticas precisas e não intuitivas como, por exemplo, vetores de posição, tangentes e mistos.

Muitos destes problemas podem ser resolvidos utilizando-se uma extensão de curvas de Bézier para superfícies. Uma superfície de Bézier é dada por:

$$
Q(u, w)=\sum_{i=0}^{n} \sum_{j=0}^{m} B_{i, j} J_{n, i}(u) K_{m, j}(w),
$$

onde $J_{n, i}(u)$ e $K_{m, j}(w)$ são funções de base de Bernstein nas direções paramétricas de $u$ e $w$, dadas por:

$$
\begin{aligned}
J_{n, i}(u) & =\left(\begin{array}{c}
n \\
i
\end{array}\right) u^{i}(1-u)^{n-i}, \\
K_{m, j}(w) & =\left(\begin{array}{c}
m \\
j
\end{array}\right) w^{j}(1-w)^{m-j},
\end{aligned}
$$

$\operatorname{com}\left(\begin{array}{c}n \\ i\end{array}\right)=\frac{n !}{i !(n-i) !}$ e $\left(\begin{array}{c}m \\ j\end{array}\right)=\frac{m !}{j !(m-j) !}$.

Aqui, os $B_{i, j}$ são os vértices da malha poligonal que define a superfície (Figura 3.). Sendo $N$ e $M$ os números de vértices da malha poligonal nas direções de $u$ e $w$, respectivamente, então os índices $n$ e $m$ são dados por: $n=N-1$ e $m=M-1$.

Para superfícies quadriláteras, a malha poligonal deve ser topologicamente retangular, isto é, deve ter o mesmo número de vértices em cada linha poligonal.

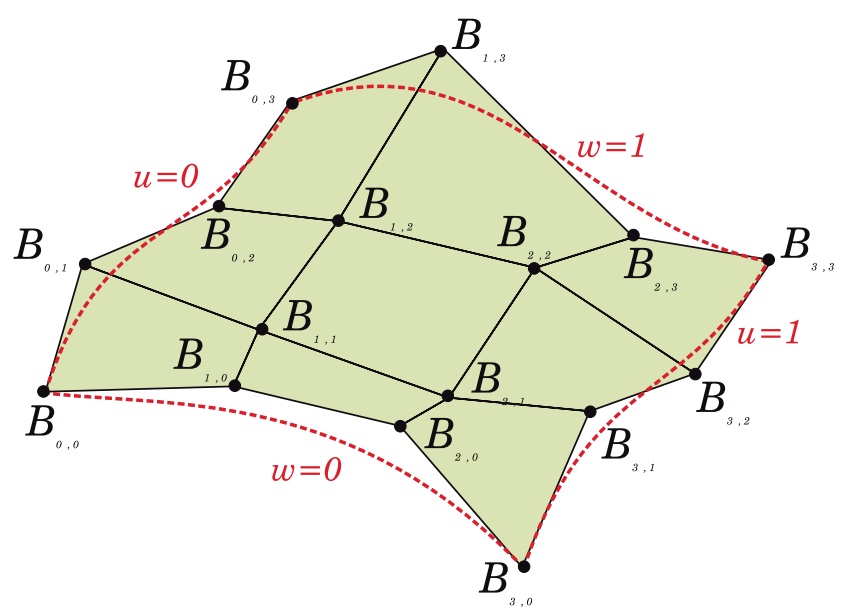

Figura 3: Malha poligonal que define a superfície de Bézier. 
Devido ao fato de superfícies de Bézier utilizarem funções de base de Bernstein como funções de combinação, muitas propriedades destas superfícies são conhecidas, como por exemplo:

- o grau da superfície em cada direção paramétrica é um a menos que o número de vértices em cada direção;

- a continuidade da superfície em cada direção paramétrica é dois a menos que o número de vértices em cada direção;

- a superfície geralmente segue o formato da malha poligonal;

- somente os pontos extremos (de canto) da superfície e da malha poligonal são coincidentes;

- a superfície está contida no fecho convexo da malha poligonal;

- a superfície é invariante sob transformações afins.

A forma matricial da equação (3.1) é dada por:

$$
Q(u, w)=[U][N][B][M]^{T}[W]
$$

onde

$$
\begin{aligned}
& {[U]=\left[\begin{array}{llll}
u^{n} & u^{n-1} & \cdots & 1
\end{array}\right],} \\
& {[W]=\left[\begin{array}{llll}
w^{m} & w^{m-1} & \cdots & 1
\end{array}\right]^{T},} \\
& {[B]=\left[\begin{array}{ccc}
B_{0,0} & \cdots & B_{0, m} \\
\vdots & \ddots & \vdots \\
B_{n, 0} & \cdots & B_{n, m}
\end{array}\right] \text {, }} \\
& {[N]=\left[\begin{array}{cccc}
\left(\begin{array}{c}
n \\
0
\end{array}\right)\left(\begin{array}{c}
n \\
n
\end{array}\right)(-1)^{n} & \left(\begin{array}{l}
n \\
1
\end{array}\right)\left(\begin{array}{c}
n-1 \\
n-1
\end{array}\right)(-1)^{n-1} & \cdots & \left(\begin{array}{c}
n \\
n
\end{array}\right)\left(\begin{array}{c}
n-n \\
n-n
\end{array}\right)(-1)^{0} \\
\left(\begin{array}{c}
n \\
0
\end{array}\right)\left(\begin{array}{c}
n \\
n-1
\end{array}\right)(-1)^{n-1} & \left(\begin{array}{c}
n \\
1
\end{array}\right)\left(\begin{array}{c}
n-1 \\
n-2
\end{array}\right)(-1)^{n-2} & \cdots & 0 \\
\vdots & \vdots & \vdots & \vdots \\
\left(\begin{array}{c}
n \\
0
\end{array}\right)\left(\begin{array}{c}
n \\
1
\end{array}\right)(-1)^{1} & \left(\begin{array}{c}
n \\
1
\end{array}\right)\left(\begin{array}{c}
n-1 \\
0
\end{array}\right)(-1)^{0} & \cdots & 0 \\
\left(\begin{array}{l}
n \\
0
\end{array}\right)\left(\begin{array}{c}
n \\
0
\end{array}\right)(-1)^{0} & 0 & \cdots & 0
\end{array}\right] \text {, }}
\end{aligned}
$$

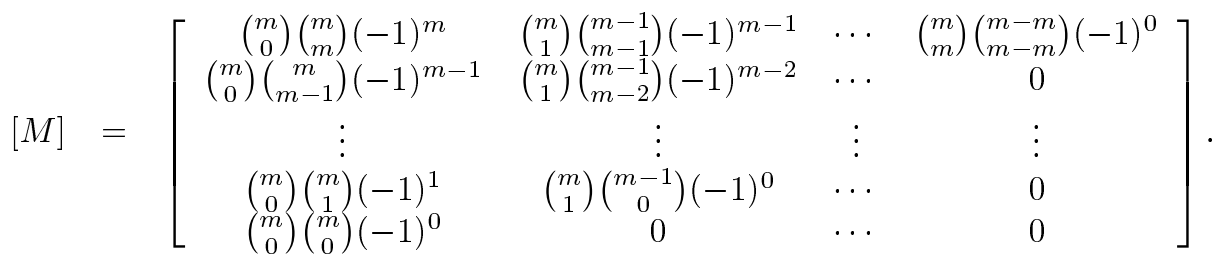


Por exemplo, para uma superfície de Bézier bicúbica $(4 \times 4)$, a equação $(3.2)$ se reduz a

$$
\begin{aligned}
Q(u, w)= & {\left[\begin{array}{llll}
u^{3} & u^{2} & u & 1
\end{array}\right]\left[\begin{array}{cccc}
-1 & 3 & -3 & 1 \\
3 & -6 & 3 & 0 \\
-3 & 3 & 0 & 0 \\
1 & 0 & 0 & 0
\end{array}\right] } \\
& \times\left[\begin{array}{lllll}
B_{0,0} & B_{0,1} & B_{0,2} & B_{0,3} \\
B_{1,0} & B_{1,1} & B_{1,2} & B_{1,3} \\
B_{2,0} & B_{2,1} & B_{2,2} & B_{2,3} \\
B_{3,0} & B_{3,1} & B_{3,2} & B_{3,3}
\end{array}\right]\left[\begin{array}{cccc}
-1 & 3 & -3 & 1 \\
3 & -6 & 3 & 0 \\
-3 & 3 & 0 & 0 \\
1 & 0 & 0 & 0
\end{array}\right]\left[\begin{array}{c}
w^{3} \\
w^{2} \\
w \\
1
\end{array}\right] .
\end{aligned}
$$

As derivadas de uma superfície de Bézier são obtidas pela diferenciação formal da equação (3.1) ou (3.2). Tomando-se a equação (3.1), as primeiras e segundas derivadas paramétricas são:

$$
\begin{aligned}
Q_{u}(u, w) & =\sum_{i=0}^{n} \sum_{j=0}^{m} B_{i, j} J_{n, i}^{\prime}(u) K_{m, j}(w), \\
Q_{w}(u, w) & =\sum_{i=0}^{n} \sum_{j=0}^{m} B_{i, j} J_{n, i}(u) K_{m, j}^{\prime}(w), \\
Q_{u w}(u, w) & =\sum_{i=0}^{n} \sum_{j=0}^{m} B_{i, j} J_{n, i}^{\prime}(u) K_{m, j}^{\prime}(w), \\
Q_{u u}(u, w) & =\sum_{i=0}^{n} \sum_{j=0}^{m} B_{i, j} J_{n, i}^{\prime \prime}(u) K_{m, j}(w), \\
Q_{w w}(u, w) & =\sum_{i=0}^{n} \sum_{j=0}^{m} B_{i, j} J_{n, i}(u) K_{m, j}^{\prime \prime}(w),
\end{aligned}
$$

onde o apóstrofo denota a diferenciação em relação à sua respectiva variável. Para $J_{n, i}(u)$, por exemplo, as derivadas são:

$$
\begin{aligned}
J_{n, i}^{\prime}(u) & =\frac{(i-n u)}{u(1-u)} J_{n, i}(u) \\
J_{n, i}^{\prime \prime}(u) & =\frac{(i-n u)^{2}-n u^{2}-i(1-2 u)}{u^{2}(1-u)^{2}} J_{n, i}(u) .
\end{aligned}
$$

\section{Superfícies B-Spline}

Sendo uma extensão natural de superfícies de Bézier, uma superfície B-Spline é dada por:

$$
Q(u, w)=\sum_{i=1}^{n+1} \sum_{j=1}^{m+1} B_{i, j} N_{i, k}(u) M_{j, l}(w),
$$


onde $N_{i, k}(u)$ e $M_{j, l}(w)$ são funções de base $B$-Spline nas direções paramétricas de $u$ e $w$, respectivamente, dadas por:

$$
\begin{aligned}
& N_{i, 1}(u)=\left\{\begin{array}{l}
1, \quad \text { se } x_{i} \leq u \leq x_{i+1} \\
0, \quad \text { caso contrário }
\end{array}\right. \\
& N_{i, k}(u)=\frac{\left(u-x_{i}\right) N_{i, k-1}(u)}{x_{i+k-1}-x_{i}}+\frac{\left(x_{i+k}-u\right) N_{i+1, k-1}(u)}{x_{i+k}-x_{i+1}}
\end{aligned}
$$

e, ainda,

$$
\begin{aligned}
& M_{j, 1}(w)=\left\{\begin{array}{l}
1, \quad \text { se } y_{j} \leq w \leq y_{j+1} \\
0, \quad \text { caso contrário }
\end{array}\right. \\
& M_{j, l}(w)=\frac{\left(w-y_{j}\right) M_{j, l-1}(w)}{y_{j+l-1}-y_{j}}+\frac{\left(y_{j+l}-w\right) M_{j+1, l-1}(w)}{y_{j+l}-y_{j+1}} .
\end{aligned}
$$

Aqui, os $B_{i, j}$ são os vértices da malha poligonal que definem a superfície. Novamente, para superfícies quadriláteras, a malha poligonal deve ser topologicamente retangular. Sendo $N$ e $M$ os números de vértices da malha poligonal nas direções de $u$ e $w$, respectivamente, então os índices $n$ e $m$ são dados por: $n=N-1$ e $m=M-1$.

Os valores $x_{i}$ e $y_{j}$ são obtidos a partir dos vetores de nós $[X]$ e $[Y]$, que têm uma significante influência sobre o formato da superfície $B$-Spline. Vetores abertos, periódicos e não uniformes são usados para este fim (ver mais detalhes em [1] e [5]).

Devido ao fato de funções de base $B$-Spline serem usadas para descrever as curvas de contorno e as funções de combinação, algumas propriedades destas superfícies são imediatamente conhecidas, como por exemplo:

- a ordem máxima da superfície em cada direção paramétrica é igual ao número de vértices da malha poligonal que a define;

- a continuidade da superfície é dois a menos que sua ordem em cada direção paramétrica, ou seja, $C^{k-2}$ e $C^{l-2}$ nas direções $u$ e $w$, respectivamente;

- a superfície é invariante sob transformações afins;

- se o número de vértices da malha poligonal é igual à ordem da superfície em cada direção paramétrica, então a superfície $B$-Spline se reduz a uma superfície de Bézier;

- a superfície está contida no fecho convexo da malha poligonal que a define. 


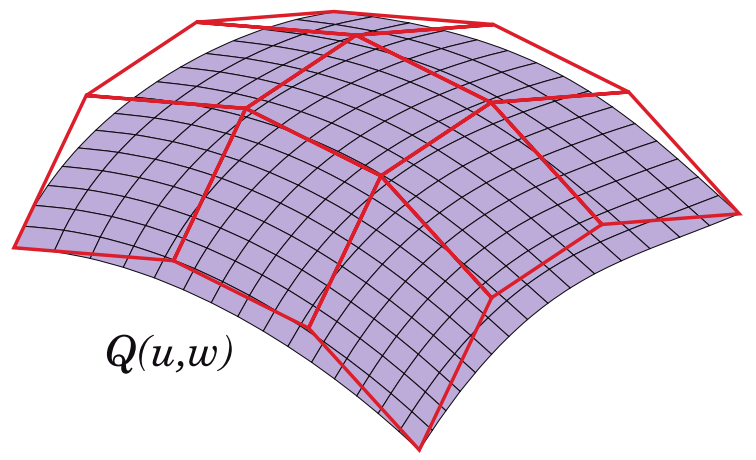

Figura 4: Superfície B-Spline e a malha poligonal que a define.

As derivadas paramétricas de uma superfície B-Spline podem ser obtidas pela diferenciação formal da equação (4.1), obtendo-se:

$$
\begin{aligned}
Q_{u}(u, w) & =\sum_{i=1}^{n+1} \sum_{j=1}^{m+1} B_{i, j} N_{i, k}^{\prime}(u) M_{j, l}(w), \\
Q_{w}(u, w) & =\sum_{i=1}^{n+1} \sum_{j=1}^{m+1} B_{i, j} N_{i, k}(u) M_{j, l}^{\prime}(w), \\
Q_{u w}(u, w) & =\sum_{i=1}^{n+1} \sum_{j=1}^{m+1} B_{i, j} N_{i, k}^{\prime}(u) M_{j, l}^{\prime}(w), \\
Q_{u u}(u, w) & =\sum_{i=1}^{n+1} \sum_{j=1}^{m+1} B_{i, j} N_{i, k}^{\prime \prime}(u) M_{j, l}(w), \\
Q_{w w}(u, w) & =\sum_{i=1}^{n+1} \sum_{j=1}^{m+1} B_{i, j} N_{i, k}(u) M_{j, l}^{\prime \prime}(w),
\end{aligned}
$$

onde o apóstrofo denota a diferenciação em relação à sua respectiva variável. Para $N_{i, k}(u)$, por exemplo, as derivadas são:

$$
\begin{aligned}
N_{i, k}^{\prime}(u)= & \frac{N_{i, k-1}(u)+\left(u-x_{i}\right) N_{i, k-1}^{\prime}(u)}{x_{i+k-1}-x_{i}} \\
& +\frac{\left(x_{i+k}-u\right) N_{i+1, k-1}^{\prime}(u)-N_{i+1, k-1}(u)}{x_{i+k}-x_{i+1}}, \\
N_{i, k}^{\prime \prime}(u)= & \frac{2 N_{i, k-1}^{\prime}(u)+\left(u-x_{i}\right) N_{i, k-1}^{\prime \prime}(u)}{x_{i+k-1}-x_{i}} \\
& +\frac{\left(x_{i+k}-u\right) N_{i+1, k-1}^{\prime \prime}(u)-2 N_{i+1, k-1}^{\prime}(u)}{x_{i+k}-x_{i+1}} .
\end{aligned}
$$




\section{Resultados}

Como resultado da teoria apresentada aqui, foram desenvolvidos e implementados, junto ao módulo de visualização de superfícies topográficas MicroDTM ([6] e [7]), algoritmos para a suavização de malhas triangulares utilizando dois métodos: superfícies de Bézier e superfícies B-Spline. Considere a malha triangular dada pela Figura 5.

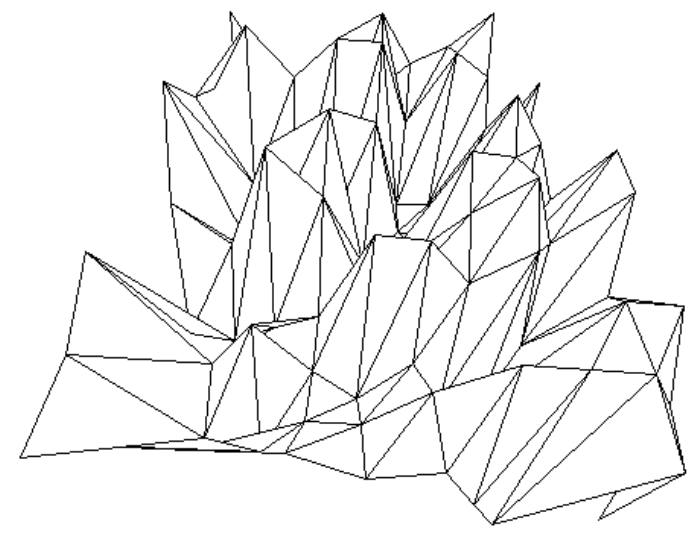

Figura 5: Malha triangular de definição.

Nas figuras seguintes, pode-se verificar as superfícies obtidas por suavização Bézier (Figura 6) e B-Spline de ordem 3 (Figura 7), com criação de 60 linhas paramétricas em ambas as direções $u$ e $w$.

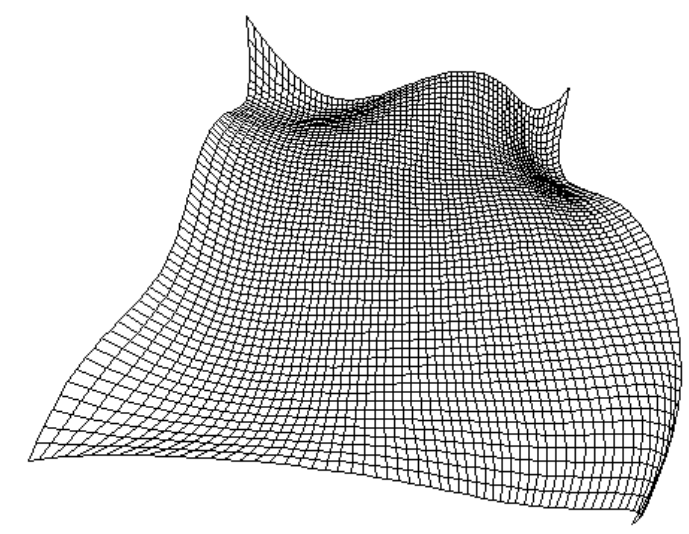

Figura 6: Superfície de Bézier gerada a partir da malha da figura anterior. 


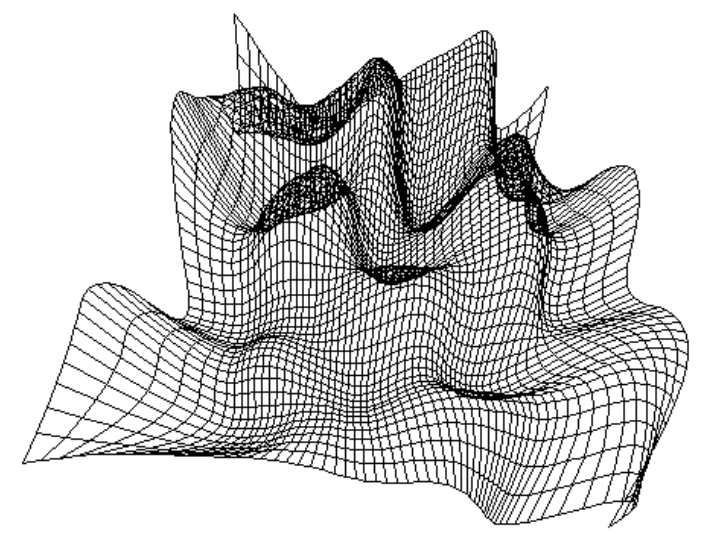

Figura 7: Superfície B-Spline de ordem 3 obtida da mesma malha.

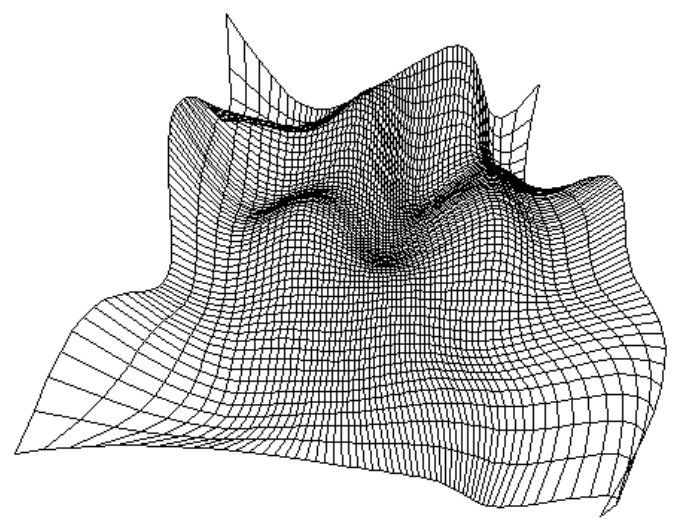

Figura 8: Superfície B-Spline de ordem 6. 
A ordem da superfície $B$-Spline muda consideravelmente a apresentação da superfície. Por exemplo, a mesma malha poligonal pode gerar uma superfície mais suave, como visto na Figura 8.

\section{Referências}

[1] G. Farin, "Curves and Surfaces for Computer Aided Geometric Design", Academic Press, 1990.

[2] L. H. Figueiredo e P. C. P. Carvalho, Introdução à Geometria Computacional, $18^{\circ}$ Colóquio Brasileiro de Matemática, IMPA, 1991.

[3] J. D. Foley, A. van Dam, S. K. Feiner and J. F. Hughe, "Computer Graphics: Principles and Pratice", Addison Wesley, 1990.

[4] G. Glaeser, "Fast Algorithms for 3D-Graphics", Springer-Verlag, 1994.

[5] D. F. Rogers and J. A. Adams, "Mathematical Elements for Computer Graphics", McGraw-Hill, 1990.

[6] F. S. Sousa M. Meneguette Jr., Apresentação computacional de objetos 3D em vistas paralela e perspectiva, com ênfase a superfícies topográficas e isolinhas, XXI CNMAC, setembro, 1998.

[7] F. S. Sousa e M. Meneguette Jr., MicroDTM: Um módulo para a visualização de superfícies topográficas, I Fórum de Ciências da FCT, novembro, 1998. 
